\title{
Self-reported Occupational Risk for COVID-19 in Hospital Doctors from Black Asian \& Minority Ethnic Communities in UK
}

Indranil Chakravorty PhD FRCP*, Sunil Daga PhD MRCP*, JS Bamrah FRCPsych CBE, Geeta Menon MD FRCOpth, Subodh Dave FRCPsych MMed, Subarna Chakravorty PhD MRCPCH FRCPath, Neeraj Bhala DPhil FRCPE FRCP and Ramesh Mehta MD FRCPCH OBE ${ }^{*}$ combined first authors BAPIO Institute for Health Research, Bedford, UK Correspondence to - admin@bapio.co.uk

\section{Abstract}

Emerging data from COVID-19 pandemic shows a trend for increased risk for healthcare workers in the UK, compared to other countries. In addition, there is a disproportionately high risk observed in healthcare workers from Black, Asian \& Minority Ethnic backgrounds. This high risk is independent of biological or demographic variables. This paper presents sub-analysis of a larger survey of healthcare workers, particularly describing possible occupational risk of COVID-19 in a subset of doctors in UK hospitals from a BAME background. The results show higher rates of inability to access personal protection or comply with social distancing. The inability to self-isolate was associated with a $1.7 x$ higher risk of COVID-19. The results of this survey suggest further research is needed to explore and understand institutional factors that may explain excess risks to BAME hospital doctors.

Key words:

COVID-19, BAME, Healthcare workers, Personal Protective Equipment, Social distancing

Cite as: Chakravorty, I., Daga, S., Bhamra, J.S., Menon, G., Dave, D., Chakravorty, S., Bhala, N., Mehta, R. (2020) Self-reported Occupational Risk for COVID-19 in Hospital Doctors from Black Asian \& Minority Ethnic Communities in UK. The Physician 6(1); v1 pre-print DOI: 10.38192/1.6.1.9

\section{Background}

By the nature of their work in caring for patients, healthcare workers (HCWs) remain at the forefront of the risks of acquiring COVID-19. Compared to published data from China, there is a high prevalence of HCWs being infected in France, Italy, Spain and USA during the current pandemic (1-3) Analysis of hospital admission data in the UK, clearly demonstrates a higher risk of COVID-19 severe disease and death in Black, Asian and minority ethnic (BAME) background being affected in the UK and USA (4). To date the mortality among UK HCWs has surpassed that of UK soldiers in the Iraq war (5). Further breakdown of data reveals a higher risk of severe COVID-19 disease and death among BAME HCWs than their Caucasian 
peers (6). UK National Health Service employers are employing potential strategies to identify and shield staff at risk.

The potential causes of this excess risk of death or disease among UK HCWs remains poorly understood. Population level data analysis suggests that certain inherent or biological variables such as age, sex, body mass index, concurrent cardiovascular, diabetes or kidney disease and previous levels of activity may determine outcomes. However, when such potential biological contributory factors are accounted for in multivariate analysis, BAME background appears to remain an independent risk (7). Exploring nonbiological factors, it is clear that BAME HCWs are a widely diverse group in religious, socio-cultural aspects as well as perceptions and beliefs (8-9). Yet the observed data demonstrates a unique commonality in the severity of outcomes within this pandemic, which cannot be explained away easily. A deeper exploration of differential experience of BAME HCWs in the UK NHS may be necessary within the context of their shared occupational experience and perceptions.

The British Association of Physicians of Indian Origin, in its 25 years of experience in working with diverse groups of HCWs, predominantly doctors from the Indian subcontinent through engagement with its national membership and their widening social networks provided the perfect vehicle for exploring the risks and perceptions of BAME HCWs. In a national online survey of a wide range of HCWs across different clinical settings, there were clear themes demonstrating perceptions of enhanced risks and behaviours pertaining to profession, clinical exposure, personal protection and even organisational issues (7). Published mortality figures among HCWs showed that as a group, $95 \%$ of doctors who had lost their lives in the current pandemic, were from a BAME background (6). In addition, within the heterogeneity of the survey respondents, hospital doctors from a BAME background provided a subset with the most common shared experience and a group that was most likely to provide an insight in understanding the excess mortality. Hence, this group was chosen for a subset analysis.

\section{Methods}

The components of this survey were conceived through engagement with an active focus group (BAPIO Think Tank) composed of 67 members of BAPIO. The investigators from the BAPIO Institute of Health Research then designed the survey questions by expert consensus. The anonymised, cross-sectional, online survey was then distributed by email links to all members of BAPIO and through social networks for a period of 2 weeks in April 2020. Results were collected online, using Google forms. Details of the survey components, questions and results are published (7). A subset of BAME hospital doctors were selected from the survey respondents during this analysis. Non-parametric tests including Chi square tests and multivariate logistic regression analysis was applied to adjust for potential contributory variables in generating odds ratios, using SPSS statistical software (SPSS v26, IBM Inc., USA).

Primary outcome - Self-reported, point prevalence of (i) access to personal protective equipment (PPE), (ii) ability to comply with social distancing guidance during work/ commuting, (iii) ability to self-isolate if at enhanced risk and predicted risk of COVID-19 (defined as positive viral PCR swab test result or selfisolating due to classical symptoms based on published Public Health England and NHS guidance.

\section{Results}

There were 1243 BAME hospital doctors in the cohort with a distributed age range (Figure 1). Majority of respondents were middle-aged (40-60 years of age) and $33 \%$ had one or more comorbidities 
(cardiovascular, hypertension, cerebrovascular, kidney, diabetes, chronic lung disease and mental health issues (Figure 2).

Figure 1: Histogram showing the age ranges for BAME doctors.

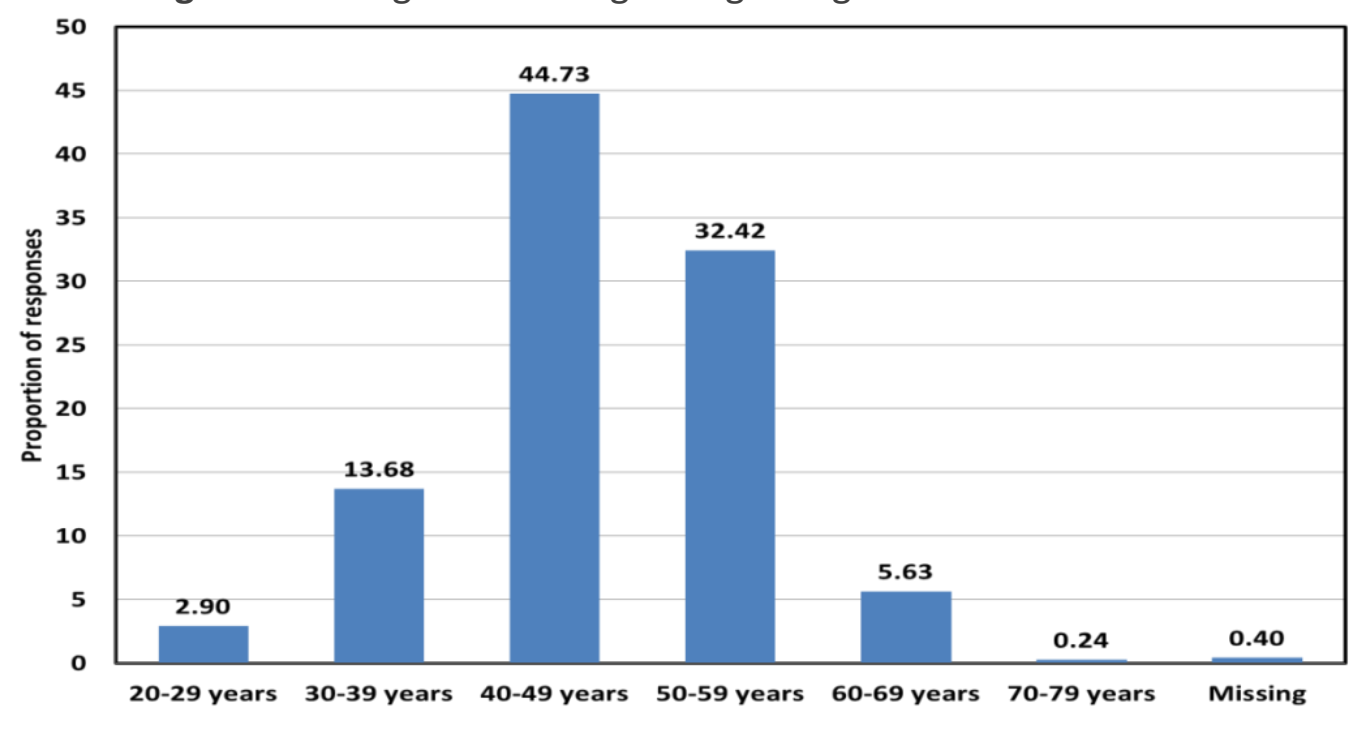

Figure 2: Histogram showing the proportion of BAME doctors with comorbidities.

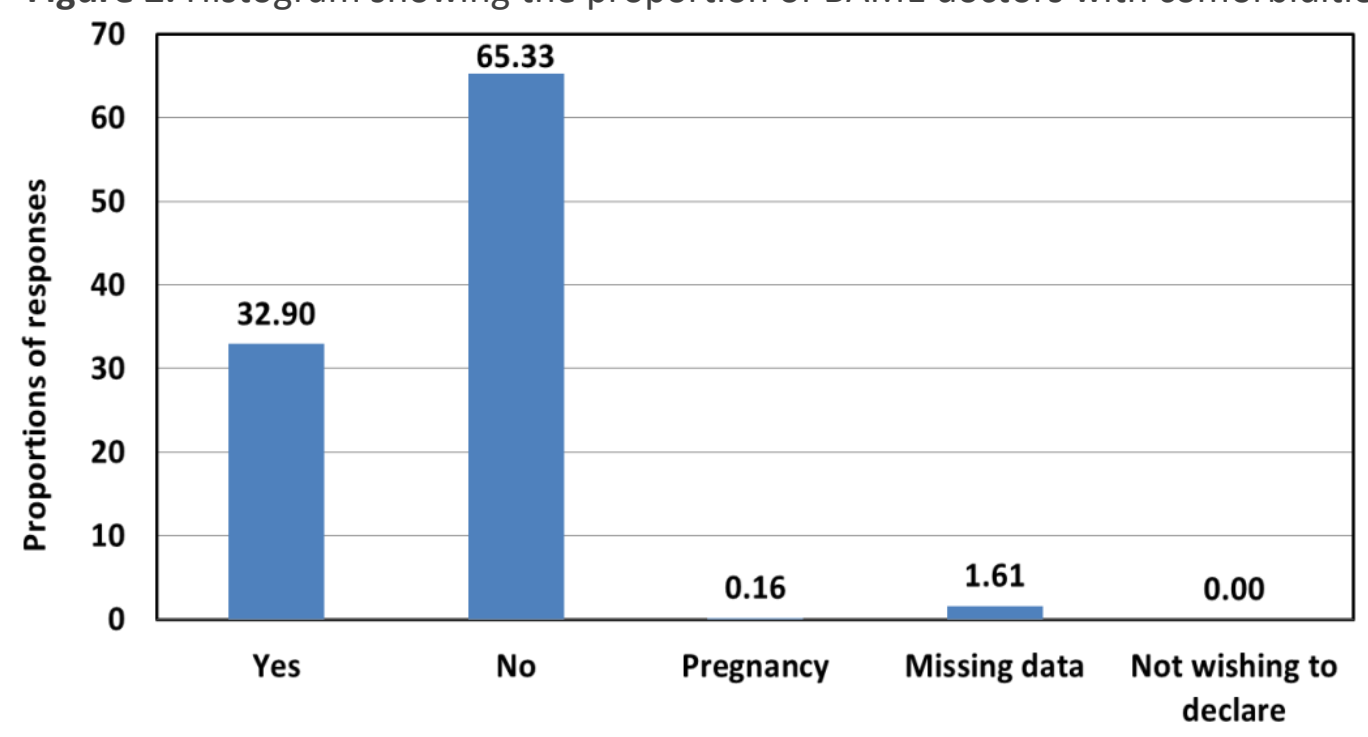

Occupational measures to reduce risk:

Only $22 \%$ have reported access to or compliance with recommended / appropriate PPE (Figure 3). Similarly, only $22 \%$ of respondents were able to fully comply with social distancing (SD) guidance at work. 
Around $35 \%$ of hospital doctors reported that it was not practical, given the nature of the clinical setting, to comply with SD at work and 36\% reported not being able to fully comply with SD guidance (Figure 4). There were $23 \%$ of respondents who reported inability to self-isolate when they perceived there was a personal / family risk of COVID-19 infection (Figure 5).

Figure 3: Pie charting showing Personal protective equipment (PPE) at work place

: 2 PPE has always been available and appropriate :- I have been restricted/ reprimanded from wearing PPE DPE has been always available but not always appropriate for my role Missing Data

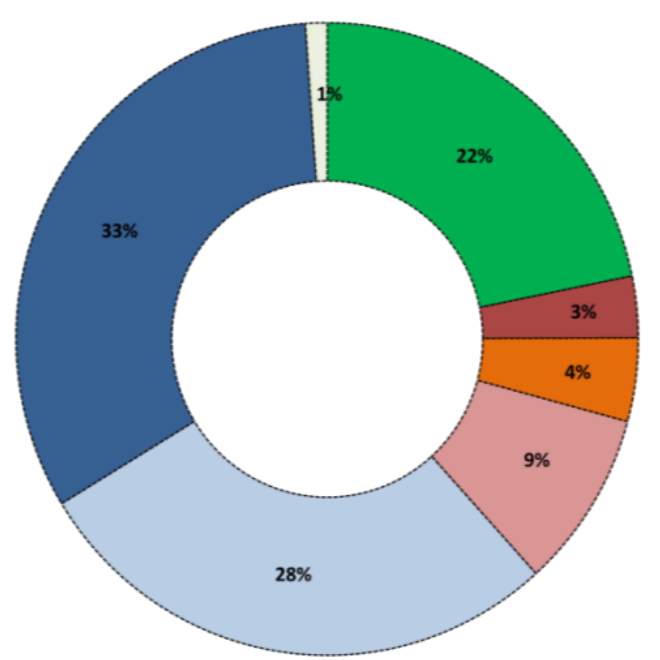

No PPE available for my role (at any time during this pandemic) DPPE has been inappropriate for my role w PPE has been in short supply/ restricted

Figure 4: Pie chart showing ability to comply with Social Distancing (SD)

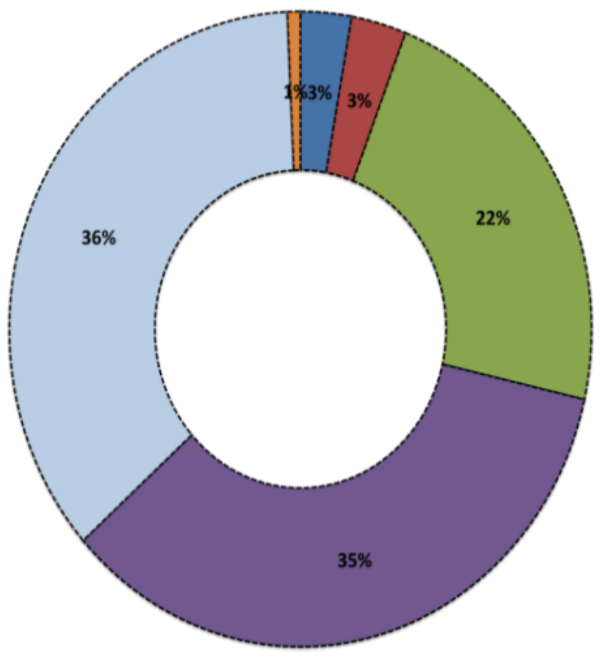

Es I cannot fully comply with SD guidance during commuting

EI I am able to comply fully with SD guidance

aI I am not able to comply fully with SD guidance w I am working away from direct patient contact due to health/ family reason $\mathrm{E}$ It is not practical to expect to comply with SD at work en Missing data

Figure 5: Reported self-isolation and risks to self/family 


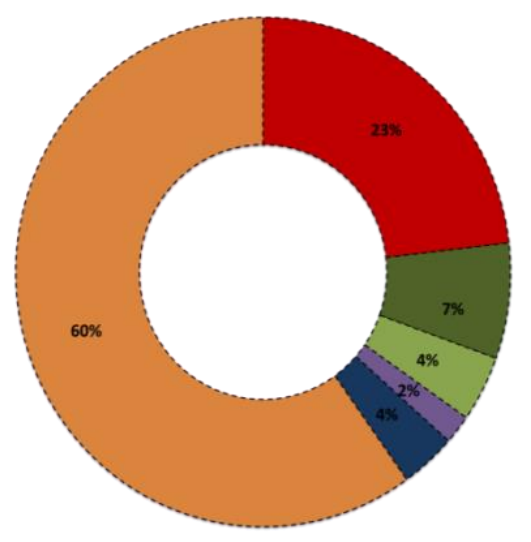

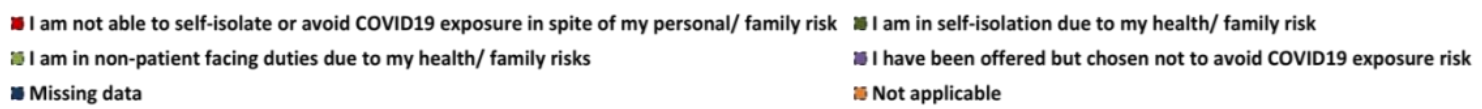

Self-reported COVID19 illness and risk analysis:

Around $5 \%$ of the hospital doctors were in shielding or proactively avoiding exposure to COVID-19 and $18 \%$ reported a confirmed positive viral PCR confirming COVID-19 or were self-isolating at home due to symptoms of COVID19 (Figure 6). The odds ratio for BAME hospital doctors having COVID-19 diagnosis or symptoms associated with COVID-19 (where testing was not available), when not being able to self-isolate due to personal risk was 1.73 (confidence interval 1.19-2.52, $p=0.004$ ) after adjusting for age and comorbidities.

Figure 6: Pie chart showing COVID19 related illness in BAME hospital doctors

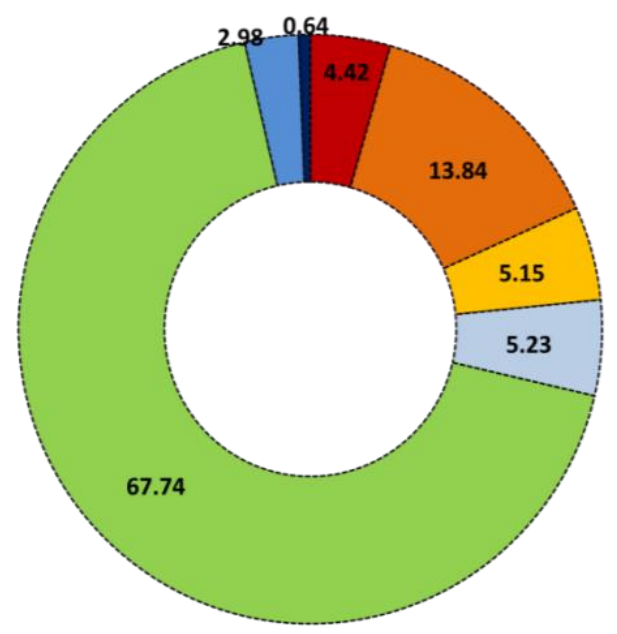

Confirmed diagnosis of COVID19 from swab test C COVID19 family contact- self isolating No symptoms or diagnosis of COVID19 Missing data we Suspected COVID19/ self-isolating In shielding/ pro-actively avoiding exposure to COVID19 Not applicable

\section{Discussions}




\section{What does the data mean?}

The results of this subset analysis of hospital doctors from a BAME background was conducted to explore the likely causes of disproportionate representation of this group in adverse outcomes in almost all recent published data in the UK. The BAPIO online survey of healthcare workers captured an overwhelming majority of hospital doctors from BAME backgrounds, so offered us a unique opportunity to explore the risks and potential vulnerability in this group (7). Our data showed that nearly 1 out of $5(18.26 \%)$ had COVID-19 or had symptoms requiring quarantine. This figure is higher than that reported for prevalence of infection in HCWs from similar surveys $(1,10-11)$.

We then explored the potential confounding factors that might explain this risk. Looking at personal protective equipment, we found almost half of BAME hospital doctors did not report that they had access to PPE as per PHE guidance. An overwhelming 3 out 4 reported not being able to comply with social distancing guidance when at work or commuting for work. These proportions appear to be higher than reported in other surveys $(10,11)$. What appeared to be most concerning was our analysis suggesting that hospital doctors from a BAME background, who were unable to comply with self-isolation guidance (based on a personal risk characteristic for COVID-19), were 1.7x more likely to have COVID-19; even when adjusted for age and comorbidities.

There are potential limitations to the extent of extrapolation of the conclusions drawn from this survey when compared to the population of BAME doctors in the UK health service. As explored in our original paper (7), these include a self-selection bias, the dependence on the accuracy of the respondents' selfdeclaration of their profession and circumstances, the perception bias for reporting issues with PPE, social distancing and the diagnosis of COVID-19. These limitations are mitigated to an extent by the nature of the distribution of the questionnaire through membership databases and bona-fide professional social networks. Clearly, the results of a survey such as this and its subset analysis must be interpreted with caution, is unlikely to be generalisable beyond the defined group and merely suggests that urgent further well-designed, controlled studies are necessary.

\section{What should be done?}

Firstly more studies are needed urgently to confirm the 'vulnerability or health risk' for BAME doctors in the COVID-19 pandemic by comparing a case-control study with non-BAME doctors and perhaps extended to all health care workers who are at the forefront of the COVID-19 response. The scientific exploration of risks must not shy away from examining uncomfortable topics that might constitute independent variables including individual and organisational factors. Discrimination has been linked to poorer health outcomes (12) and this will be a valid variable to examine amongst others.

Secondly, until further robust research results are available, BAME doctors with vulnerability should be offered/ encouraged to comply with strategies for avoidance of exposure. These may be in the form of redeployment away from high risk areas, with limitation to direct patient contact or access to enhanced PPE as available in aerosol generating clinical settings.

And, finally these results pooled with emerging clinical data should be computed to create a risk stratification tool so HCWs with known vulnerability (i.e. ethnicity) can be offered appropriate safety and deployment. It is encouraging to note recent guidance on risk assessments of BAME healthcare workers 
at workplace in NHS (13), though a validated culturally appropriate risk assessment tool is urgently needed accounting for socio-cultural factors specific for BAME communities.

\section{Author's contribution and conflict of interest statements}

The authors have no declared conflict of interest pertaining to this study. Indranil Chakravorty (IC), Sunil Daga (SKD), Geeta Menon (GM), Subodh Dave (SD), Subarna Chakravorty (SC), Neeraj Bhala (NB), Ramesh Mehta (RM) \& JS Bamrah (JSB) are honorary members of BAPIO Institute for Health Research, Bedford, UK. BAPIO is a national professional body of multi-professional health care workers in the UK since 1996 (https://www. bapio.co.uk/). Individual contributions are study design (IC, SKD, JSB \& SC); analysis (IC \& SKD); interpreting the results and writing the manuscript (all).

\section{Acknowledgement:}

The authors wish to thank the staff at BAPIO Head Office, particularly Sneha Deshpande and to all members of BAPIO Think Tank who had helped to distribute the survey.

\section{References}

1. COVID-19: protecting health-care workers. Lancet 2020, Volume 395, ISSUE 10228, P922, March 2020

2. https://news.sky.com/story/coronavirus-half-of-a-e-staff-at-one-hospital-in-wales-have-covid19-reveals-medic-11972384

3. https://www.telegraph.co.uk/news/0/nhs-died-coronavirus-frontline-workers-victims/

4. https://www.ons.gov.uk/peoplepopulationandcommunity/birthsdeathsandmarriages/deaths/ar ticles/coronavirusrelateddeathsbyethnicgroupenglandandwales/2march2020to10april2020

5. Exclusive: deaths of NHS staff from covid-19 analysed; HSJ April 2020

6. ICNARC Case Mix Programme Database COVID19 Report; April 2020

7. Chakravorty, I., Daga, S., Dave, S., Chakravorty, S., Menon, G., Bhala, N., Mehta, R., \& Bamrah, J. (2020). An Online Survey of Healthcare Professionals in the COVID-19 Pandemic in the UK: : Perceptions of Risk Factors. SUSHRUTA Journal of Health Policy \& Opinions, 13(2). https://doi.org/10.38192/13.2.9

8. The COVID-19 Pandemic: a Call to Action to Identify and Address Racial and Ethnic Disparities; J Racial Ethn Health Disparities. 2020 Apr 18

9. Ethnicity and COVID-19: an urgent public health research priority; The Lancet April 2020.

10. https://www.bma.org.uk/news-and-opinion/bma-survey-finds-doctors-lives-still-at-risk-despitegovernment-pledges-on-ppe

11. https://www.rcseng.ac.uk/news-and-events/news/archive/ppe-and-testing-covid-surveyresults/

12. Racism and Health: Evidence and Needed Research; Annual Review of Public Health 2019 https://www.hsj.co.uk/workforce/revealed-the-nhs-plan-to-protect-bame-staff-from-covid19/7027571.article 